\title{
Study on He Hong's Dongba Manuscripts Collected by American Harvard-Yenching Library
}

\author{
Xiaoliang Li \\ The Institute of Chinese Language and Documents, Southwest University, Chongqing, China \\ Email: longer_2411363@163.com
}

Received November 26 ${ }^{\text {th }}$, 2013; revised December 24 $4^{\text {th }}$, 2013; accepted January $19^{\text {th }}, 2014$

\begin{abstract}
Copyright (c) 2014 Xiaoliang Li. This is an open access article distributed under the Creative Commons Attribution License, which permits unrestricted use, distribution, and reproduction in any medium, provided the original work is properly cited. In accordance of the Creative Commons Attribution License all Copyrights (C) 2014 are reserved for SCIRP and the owner of the intellectual property Xiaoliang Li. All Copyright @ 2014 are guarded by law and by SCIRP as a guardian.
\end{abstract}

\begin{abstract}
American Harvard-Yenching Library has uploaded its Dongba manuscripts of Naxi on the official website Most of the Dongba manuscripts collection is featured with older version, good appearance, fluent and elegant writing, and the portraits are true to life. Therefore, Harvard Dongba manuscripts collections are definitely rare collections. Some of these manuscripts were written by He Hong, who was one of well-known Dongba, native Naxi priests, in Baisha, Lijiang. This paper focuses on several aspects of the title pages, writing style, postscript of He Hong's manuscripts in order that we can understand the characteristic of the Dongba manuscripts of Baisha.
\end{abstract}

Keywords: Harvard-Yenching Library; He Hong; Dongba Manuscripts

\section{Introduction}

The Naxi (also written Na-khi or Mo-so) is an ethnic minority in the northwestern part of Yunnan Province in China, near the borders of Tibet and Burma. Their pictographic Dongba (also written dto-mba or tomba) script is the only remaining living pictographic language today. It is called the Dongba script because it is primarily utilized by Dongba or priests when they carry out their different ceremonies, rituals and exorcisms. Dongba script was written on Dongba manuscripts. The actual story of the Dongba manuscripts began way back in 1867 when the French missionary, Pere Desgodins, sent an 11 pages copy of Dongba manuscripts to Paris. Subsequently, more Dongba manuscripts were brought back to Europe or America by travelers in Yunnan and they were discussed in terms of the development of writing (Jackson, 1989: p. 135). According to Britain scholar Anthony Jackson's survey, about ten thousand Dongba manuscripts lost overseas.

There are 598 manuscripts in Harvard-Yenching Library, and the Harvard-Yenching Library's holdings (510 from Joseph Rock and 88 from Quentin Roosevelt) were acquired by Harvard-Yenching Institute in 1945. Joseph Rock, Zhu Baotian, and $\mathrm{Li}$ Lincan edited these manuscripts respectively. Joseph Rock catalogued the Dongba manuscripts collected by himself. Li Lincan explained 21 pieces of these manuscripts and translated some manuscripts' name into Chinese. Zhu Baotian catalogued all of those manuscripts and we can check the material in his works An Annotated Catalog of Naxi Pictographic Manuscripts in the Harvard-Yenching Library. In addition, He Jiquan, Deng Zhangying and Zhang Chunfeng have studied on them respectively. He Jiquan translated the postscript of some manuscripts and determined the identity of some classics writers. Deng Zhangying and Zhang Chunfeng studied the manu- scripts stamped with two red circles. At present the Harvard-Yenching Institute, the Institute of Ethnology and Anthropology, Chinese Academy of Social Science and the Institute of Dongba Culture at the Yunnan Academy of Social Science are translating the 598 manuscripts.

According to Li Guowen (1998: p. 227), He Hong was a Dongba who lived in Baisha Town, Lijiang, during the late Qing Dynasty. He was the nineteenth generation descendants of a famous Dongba Jiuzhilao, and he is also the grandfather of $\mathrm{He}$ Cheng who was a famous Dongba during the early Republic of China. We have found that 50 manuscripts among the Harvard Dongba manuscripts collections were written by He Hong. These manuscripts are used in the ceremony for the prolongation of the life. The Naxi language pronunciation of the ceremony's title is $\mathrm{z}^{33} \mathrm{tsu}^{55}$, which means "long life connect", and a common theme is that things have been disconnected, for example heaven and earth, mountain and valley, the cardinal points, and of course longevity and the family. This ceremony is to make good this interruption of continued long life (Jackson, 1979: p. 190).

This paper focuses on several aspects of the title page, writing style, postscript of He Hong's manuscripts in order that we can understand the characteristic of the Dongba manuscripts of Baisha.

\section{Postscript of He Hong's Dongba Manuscripts}

It is the direct evidence for us to determine the identity of the writer if there is signature in the postscripts. Referring to the following postscripts, we can determine that these manuscripts are written by He Hong.

This page (Figure 1) is a postscript written in Dongba script and the translation is, "it was written by He Hong who lived in 


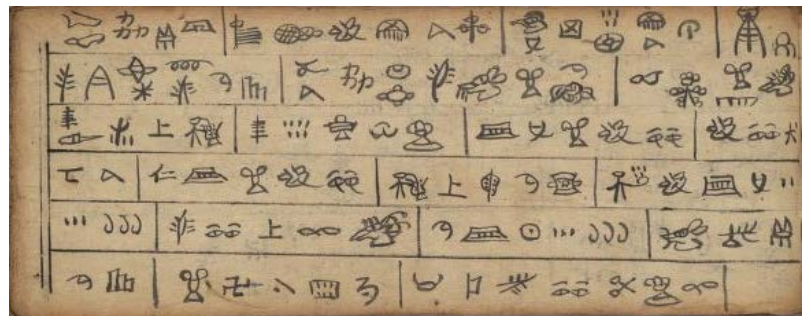

Figure 1.

D3.

Baisha countryside, be ${ }^{33} \mathrm{kv}^{33}$ village, on August 15th. There are no thousands of meters tree in the pine forest and snow mountain. In our village, there is no one that can live beyond 100 years old. In the world, holding ceremonies is hoped to prolong longevity. The insider knows the ropes, while the outsider just comes along for the ride. I have taught others what I've know. I couldn't turn to anybody for help about what I haven't known. The kind-hearted man died, but their good reputation spread the later generation.”

The symbol represents ribs, here reads ho ${ }^{21}$. The next symbol represents stomach of a ruminant, here read shu ${ }^{21}$, and the two symbols represent a person's name, He Hong.

From the second line is a postscript (Figure 2). The first 4 lines are written in Geba script which is a kind of Naxi syllabic character; the next 2 line are written in Dongba script. Translation is, "The man who was born in $\mathrm{mbu}^{33}$ tho $^{21}$ soil rabbit year wrote this manuscript in Lunar August 20th. He Hong who lived in the village of $\mathrm{be}^{33} \mathrm{kv}^{33} \mathrm{la}^{33} \mathrm{zo}^{33} \mathrm{tshu}^{33} \mathrm{~g}^{33} \mathrm{i}^{33} \mathrm{kæ}^{33}$ wrote this manuscript at the age of fifty-eight. Life always fills up with disappointment. I hope everything goes well. Good reputation will never lose. Let the priest longevity.” The symbol $\bar{\imath}$, here reads hu, and the next symbol $\overline{\bar{\nu}}$, here reads ho, and the two symbols represent a person's name, He Hong.

This postscript (Figure 3) is written in Dongba script, "The man who was born in $\mathrm{mbu}^{33}$ tho $^{21}$ soil rabbit year wrote this manuscript in Lunar March. He Hong who lived in the village of $\mathrm{be}^{33} \mathrm{kv}^{33} \mathrm{la}^{33} \mathrm{zo}^{33} \mathrm{tshu}^{33} \mathrm{~g}^{33} \mathrm{i}^{33} \mathrm{kæ}^{33}$ wrote this manuscript at the age of fifty-one. It's easier said than done. Look before leap."

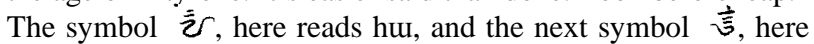
reads ho, and the two symbols represent a person's name, He Hong.

From the beginning of the second line is a postscript (Figure 4) and the translation is, "He Hong who lived in the village of $\mathrm{be}^{33} \mathrm{kv}^{33} \mathrm{la}^{33} \mathrm{zo}^{33} \mathrm{tshu}^{33} \mathrm{~g}^{33} \mathrm{i}^{33} \mathrm{kæ}^{33}$ wrote this manuscript at the age of sixty-one. May me longevity. My descents will happy

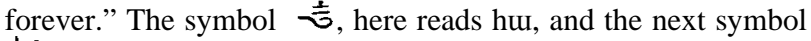
$\mathfrak{r}$, here reads ho, and the two symbols represent a person's name, He Hong.

The signatures of He Hong in the four postscripts above are different. But in the light of handwriting and pronunciation of Dongba and Geba scripts, they actually refer to the same person.

\section{Determining He Hong's Year of Birth}

About He Cheng who was He Hong's grandson, Li Guowen (1998: p. 277), “He Cheng (1879-1953) lived in Xinshan village, Baisha Xiang, Lijiang (丽江白沙乡新善行政村), who was the 21st descendant of a famous Dongba Jiuzhilao. He Hong is the grandfather of He Cheng."

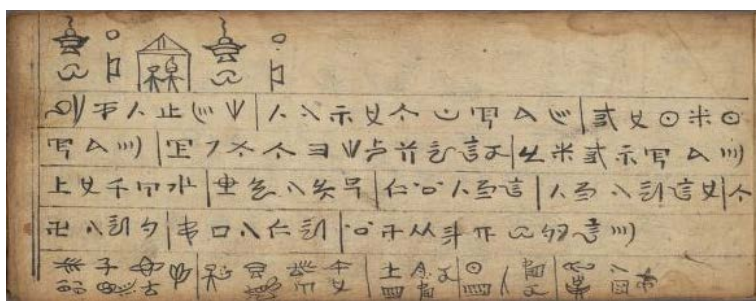

Figure 2.

D9.

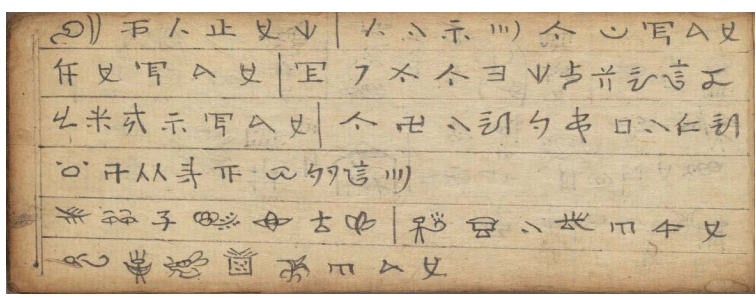

Figure 3.

D20.

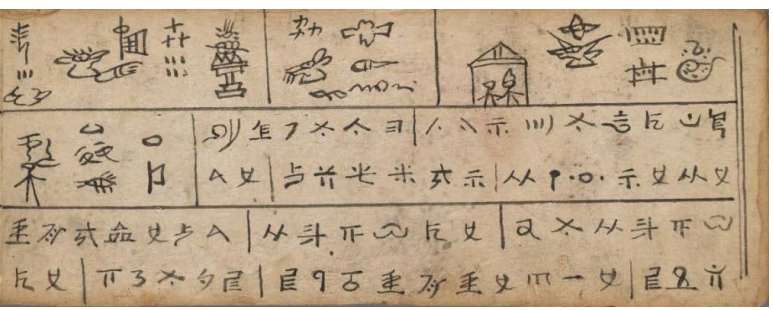

Figure 4.

D43.

From the above, He Hong may live in the late Qing Dynasty, but the accurate time is unknown. We find the key evidence in the D9 postscript, "The man who was born in mbu ${ }^{33}$ tho $^{21}$ soil rabbit year wrote this manuscript in Lunar August 20th." $\mathrm{mbu}^{33}$ tho $^{21}$ soil rabbit years may be in 1879 or 1819 . He Hong's grandson He Cheng was born in 1879. So He Hong was born in 1819.

D63 postscript can also confirm this conjecture.

Postscript (Figure 5), "Written in Guangxu three years, ox year, Lunar July 7th. Altair and lyrae are in the sky.” The first symbol To $^{3}$ represents garlic, reads $\mathrm{kv}^{33}$, here read $\mathrm{kua}^{33}$. The second symbol $\mathrm{f}^{2}$ represents wool, sheep wool, reads $\mathrm{s \tau}^{33}$, here read $\mathrm{sy}^{55}$, the two symbols represent the title of an emperor's reign, the Chinese Guangxu (光绪). The third symbol represents breath, here reads $\mathrm{sa}^{33}=$ san (三). The forth symbol $\approx$ represents eyes, here reads ni ${ }^{21}=$ nian (年). Guangxu three years is A.D.1877. So this manuscript was written in A.D. 1877.

Other postscripts with accurate time in Figure 6.

We identified He Hong's year of birth, according to the postscript of age dating, and these manuscripts can be launched in 1870 to 1886 . So He Hong at least lived between 1819 and 1886.

\section{Characteristic of He Hong's Manuscripts}

The Title Page

He Hong's manuscript uniformly bond with orange covers 


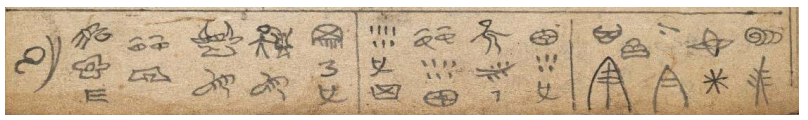

Figure 5.

D63.

\begin{tabular}{|c|c|c|c|}
\hline No. & Postscript & age & Copy time \\
\hline D67 & $\begin{array}{l}\text { The man who was born in } \mathrm{mbu}^{33} \mathrm{tho}^{21} \\
\text { soil rabbit year wrote this manuscript } \\
\text { in Lunar January } 11 \text { th. He Hong wrote } \\
\text { this manuscript at the age of fifty-one. }\end{array}$ & 51 & A.D. 1870 \\
\hline D24 & $\begin{array}{l}\text { Written in Lunar April } 9 \text { th. Writing from } \\
5 \text { a.m. to } 3 \text { p.m. He wrote this } \\
\text { manuscript at the age of fifty-eight. }\end{array}$ & 58 & A.D.1877 \\
\hline D28 & I wrote this manuscript at age of sixty-one. & 61 & A.D. 1880 \\
\hline D43 & $\begin{array}{l}\text { He Hong who lived in the village of } \\
\text { be }^{33} \mathrm{kv}^{33} \mathrm{la}^{33} \mathrm{zo}^{33} \mathrm{t}_{\mathrm{Sh}} \mathrm{u}^{33} \mathrm{ga}^{33} \mathrm{i} \mathrm{i}^{33} \mathrm{kæ}^{33} \\
\text { wrote this manuscript at the age of sixty-one. }\end{array}$ & 61 & A.D. 1880 \\
\hline D14 & I wrote this manuscript at age of sixty-seven. & 67 & A.D.1886 \\
\hline
\end{tabular}

Figure 6.

The postscripts with accurate time.

edged with blue, green or yellowish brown, and decorated on either side with striped borders, for example Figure 7.

\section{Title Area box}

The manuscripts with horizontally set titles were more beautifully ornamented. In most cases, the titles were set into oblong box with a variety of symbols on top of it. Four or six draperies were drawn around the upper part of the title box. The drawing skill of the Dongba writers of this type was more sophisticated than that of others, for example in Figures 8 and 9.

The title is started with a Dongba sitting in the stage and chanting. The majority of the manuscripts' title explain that which ceremony they can be used. He Hong's manuscripts are all used in ceremnny for the prolongation of the life, so the Dongba scripts reads $\mathrm{z}^{33} \mathrm{tsu}^{55} \mathrm{py}^{21}$. Joseph Rock recorded them as zĭ chung bpö. The name of the manuscript is followed.

Generally speaking, there is always a start symbol in the first age or passage. Different regions of Dongba will use different symbols. These symbols are mostly derived from the Tibetan, but it is also unusually personalized.

A single vertical lines was used as the inter sentence markers in the general manuscripts. Two vertical lines are used at the end of a passage. Additionally, in some postscript of the manuscripts circles separate the sentences. As shown in Figure 10.

He Hong manuscripts' columns pattern is the same with general Dongba manuscripts, which was divided into three columns per page. Only in postscript page, it would be divided into more than 3 columns, as in Figure 10.

\section{Joseph Rock's Sticker}

Each manuscript collected by Joseph Rock is marked with two stickers. One is on the cover (Sticker 1), and the other is on the back of cover (Sticker 2), as shown in Figures 11 and 12.

The content pattern of Sticker 1, ž chung bpö + number. Z $\breve{I}$

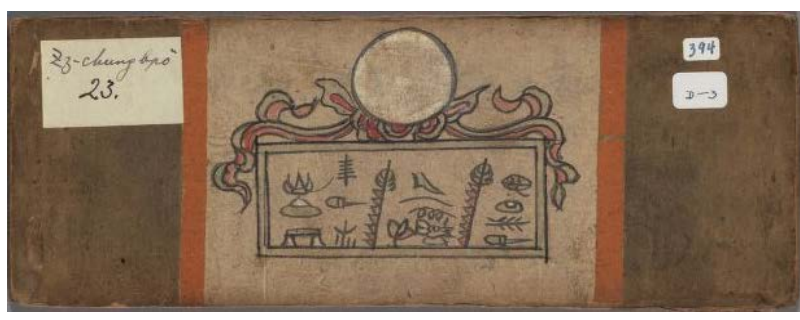

Figure 7.

D3.

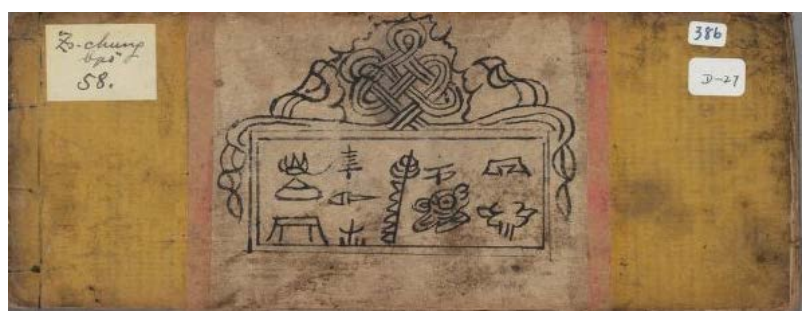

Figure 8.

D27.

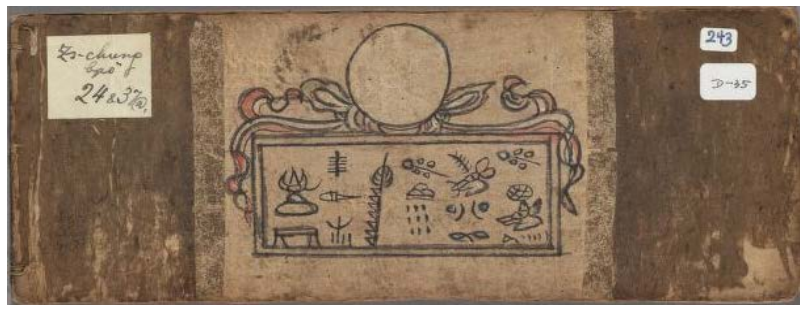

Figure 9.

D35.

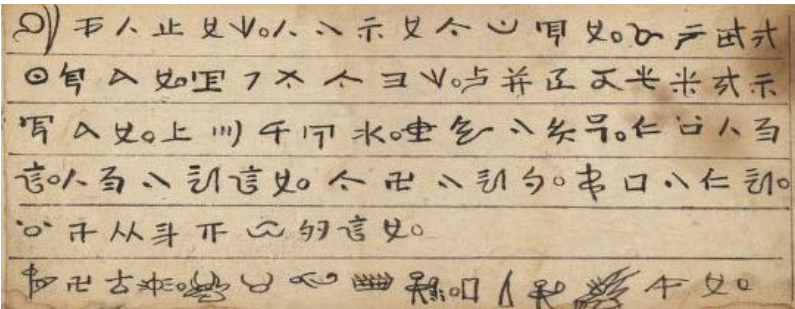

Figure 10.

D67.

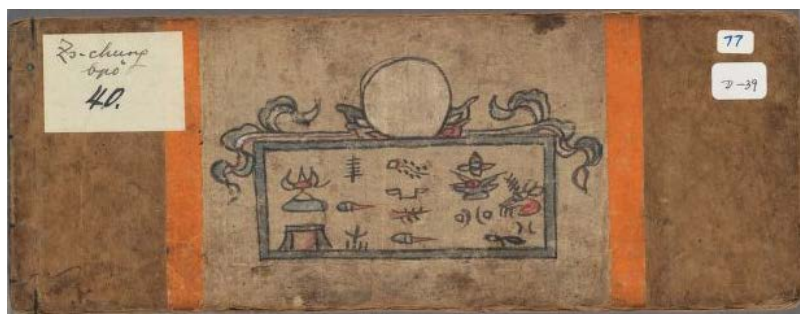

Figure 11.

D39.

chung bpö is the phonetic symbol of the ceremony for the prolongation of the life, and the number shows the kind of the ceremony. Sticker 2 was composed of manuscript's name, the phonetic symbol of name and the number which are written by 


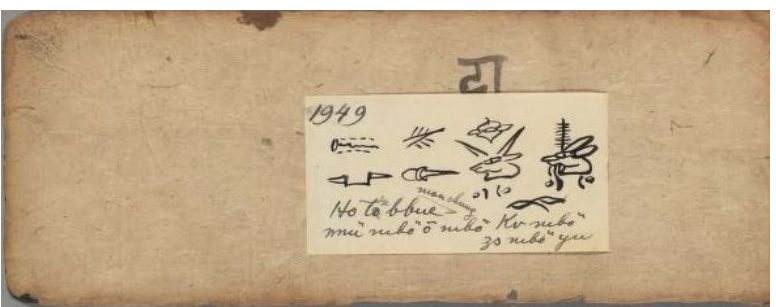

Figure 12.

D39.

Dongba script. The Sticker 2 number is commonly known as the serial number which was marked by Joseph Rock. In theory, the serial number should be unique. Harvard has 50 scriptures which copied by He Hong, 49 manuscripts' serial number is included between 1938 and 1988. We can't find No. 1987 and 1950 in Harvard's collection. We check Zhu Baotian's works An Annotated Catalog of Naxi Pictographic Manuscripts in the Harvard-Yenching Library and find that the scriptures NO. 1987 and 1950 corresponding to D10 and D15 of Zhu Baotian respectively. While the manuscripts that Harvard-yenching institute library published lack D10 and D15. We speculate that D10 and D15 both are He Hong's manuscripts. Another determined one written by He Hong is No.2061. Thus, we can conclude that this batch of scriptures was collected from the same place at the same time by Joseph Rock.

\section{Writing Style}

He Hong's writing style is obviously different from other Dongba or prests. He's script style is smaller than general Dongba script and graphic form is reserved and delicate. He Hong selected the long script spacing so that handwriting is neat and legible, for example Figure 13.

The arrangement of some postscripts' scripts was in a line across the page, reads from left to right line by line, as shown in Figure 14. The arrangement of all ritual text and some postscripts' scripts was not in a line across the page, it reads from left to right, from top to bottom, for example Figure 15.

He Hong is good at Dongba script, Geba script, Sanskrit and Chinese script.

The postscript of D9, D20, D43, D67 are written in Geba script, for example Figure 16.

The front 5 pages of Figure 17 were written in Sanskrit which are incantations. Prayers, charms, incantations were used to appease the gods or to ward off demons.

Chinese script of Figure 18, “百歲光陰爭十年, 十年更上千 秋頌, 千秋福禄綿三世, 三世相承百歲人。玉兔久成千岁藥, 桂花新放一枝香。”

By comparison with the handwriting, the poem and the test were transcribed by the same person. Even if He Hong is not the author of this poem, it can prove that He Hong's Chinese is good enough to comprehensive this poem at least.

In addition, D8 envelope have a Chinese couplet, “天青地青 道青一通, 日光月光世光萬年。” D37 envelope have a Chinese couplet, “寶矛喜见萱堂荣晚景, 金枝佇看桂龁憒秋香。”

\section{Conclusion}

\section{The Significance to Domain Division of Dongba Script}

It has long been a difficult issue for scholars to divide domain of Dongba script. The handed down manuscripts, frequent

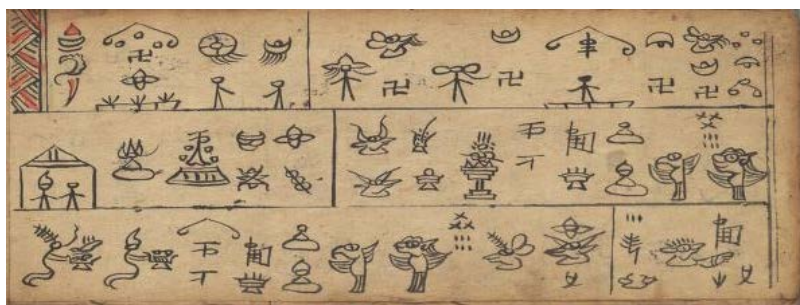

Figure 13.

D39.

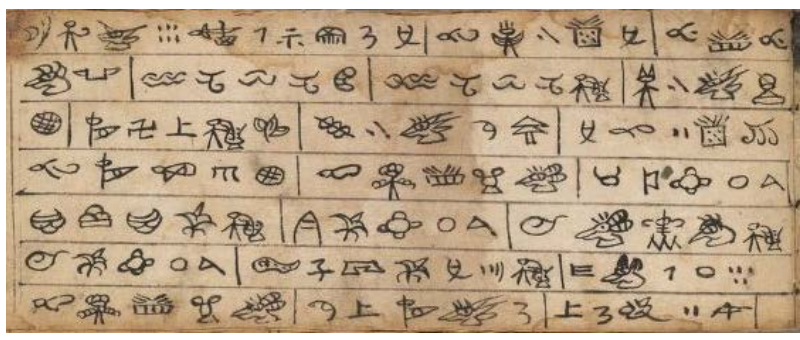

Figure 14.

D28.

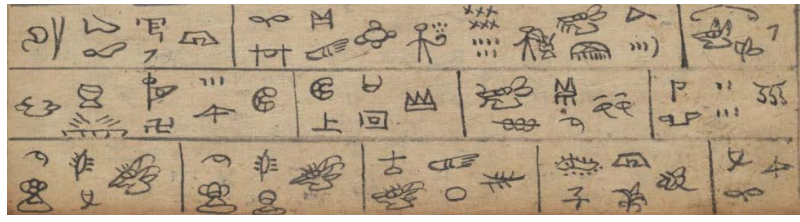

Figure 15.

D28.

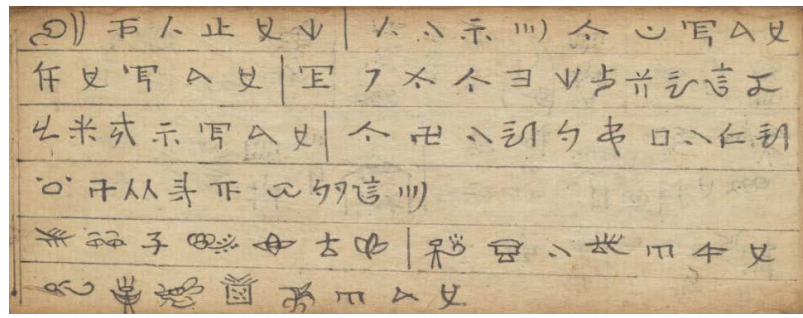

Figure 16.

D20.

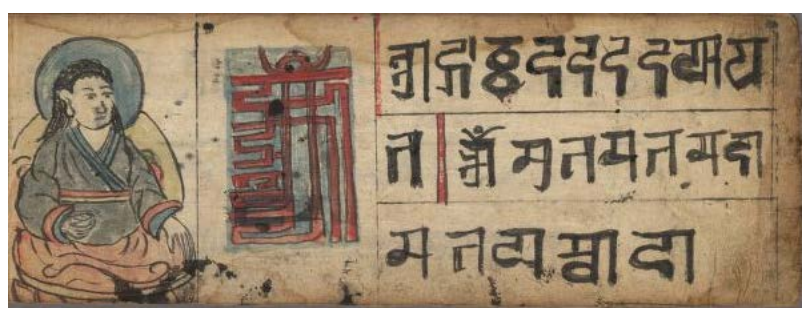

Figure 17.

D49.

usage and difficulties of long time storage make it difficult for us to see the early Dongba manuscripts. Thus, at the present stage, we can only rely on the postscripts to determine the writing age of Dongba manuscripts. The area and time of He Hong's Dongba manuscripts copy are determined, which offer us information to divide domain of Naxi manuscript. 


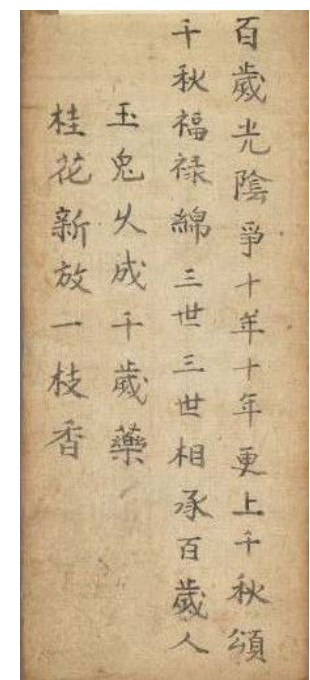

Figure 18

D32.

\section{The Significance to the Study of Naxi Culture History}

He Zhiwu and Guo Dalie referred to schools of Dongba, these Naxi Dongba in Lijiang and Zhongdian county belonged to four diffenernt schools, the schools of Baisha, Tai'an/Ludian, Baidi and Baoshan. The Baisha school include the Dongba of Lijiang plain which was and still is the economic and culture centre of the Naxi. The Dongba here produced more ritual texts and were skilful in painting and dancing than Dongba elsewhere. They used the Dongba script as well as Geba script (1985: p. 42). Influenced by the culture of Han, Dongba manuscripts in this area revolutionarily changed in writing, the title page and illustrations. As a consequence, a large number of dedicate Dongba manuscripts emerged. Whether in title page, binding and illustrations or in font and normative character usage, those Baisha Dongba manuscripts can be treated as learning models. It is of great importance to study Baisha Dongba manuscripts, which can help us to get a comprehensive understanding of Naxi culture history.

\section{The Significance to the Study of He Hong's Other Dongba Manuscripts}

The majority of the Dongba manuscripts preserved in European and American libraries or museums was collected by Joseph Rock. And lots of them lost when a boat was sunk with Rock's stuff on board, during World War II. Li Guowen has ever mentioned that He Cheng had sold all his Dongba manu- scripts to Joseph Rock. According Anthony Jackson's survey, in addition to the Library of Harvard-Yenching Institute, many other European and American libraries or museums that preserved He Hong's manuscripts. Therefore, our study on this batch of Dongba manuscripts will lay a foundation for our future research.

\section{Acknowledgements}

We are grateful to Jaan Aru for his substantial help during preparation of this report.

\section{REFERENCES}

Deng, Z. Y., \& Zhang, C. F. (2013). Study on the Dongba manuscripts stamped with two red circles of Harvard-Yenching library collection. Dongbajing Chukao: Wenxian.

He, J. Q. (2009). Study on Dongba manuscripts of American HarvardYenching library. Journal of Central University for Nationalities.

He, Z. W., \& Guo, D. L. (1985). Schools and current state of the Dongba religion. Kunming: Yunnan Renmin Press.

Jackson, A. (1965). Mo-so magical texts (Vol. 48). Bulletin John Ryland Library, Manches-ter University Press.

Jackson, A. (1979). Na-Khi religion: An analytical appraisal of the Na-khi ritual texts. The Hangue: Mouton Publisher.

Jackson, A. (1989). Naxi studies: Past, present and future. In C. Chiao, \& N. Tapp (Eds.), New Asia Academic Bulletin 8, Special Issue on Ethnicity \& Ethnic Groups in China.

Li, G. W. (1998). The mediator between people and god-Study on Dongba priests. Kunming: Yunnan Renmin Press.

Naxi manuscripts collection (2014). http://oasis.lib.harvard.edu/oasis/deliver/deepLink?_collection=oasis \&uniqueId=hyl00002

Oppiz, M., \& Hsu, E. (1998). Naxi and moso ethnography: Kin, rites, pictographs. Zurich: Volkerkundemuseum Zurich.

Rock, J. F. (1972). A Na-khi-English encyclopedic dictionary, Part II: Gods, priests, ceremonies, stars, geographical names. Rome: Instituto Italiano per il Medio ed Estremo Oriente.

Tang, G. Z. (1988). Complete poems from Song dynasty. Beijing: Zhonghua Shuju Press.

The Committee of Lijiang Naxizu Zizhixianzhi (2001). County annals of Lijiang Naxi. Kunming: Yunnan renmin Press.

The Harvard-Yenching Institute, the Institute of Ethnology and Anthropology, Chinese Academy of Social Science and the Institute of Dongba Culture at the Yunnan Academy of Social Science (2012). Naxi manuscripts collected by Harvard-Yenching Institute (pp. 1-4), Beijing: Zhongguo shehuikexue Press.

Zhu, B. T. (1995). An annotated catalog of naxi pictographic manuscripts in the Harvard-Yenching library. Harvard-Yenching Library Press. 